\title{
Quantitative Analysis of Formation of Active Avoidance Behavior in the Hippocampus Coagulated and Intact White Albino Rats
}

\author{
Sulkhan N. Tsagareli ${ }^{1}$, Nino G. Archvadze ${ }^{1}$, Otar Tavdishvili ${ }^{2}$ \\ ${ }^{1}$ Biology Department, Faculty of Exact and Natural Sciences, Ivane Javakhishvili Tbilisi State University, Tbilisi, Georgia \\ ${ }^{2}$ Department of Applied Systems for Pattern Recognition, Institute of Cybernetics, Georgian Technical University, Tbilisi, Georgia \\ Email: \{sulkhan.tsagareli, nino.archvadze\}@tsu.ge, otartavdi@yahoo.com
}

Received July 9, 2011; revised November 9, 2011; accepted November 30, 2011

\begin{abstract}
Unsupervised cluster analysis is proposed for analysis of active avoidance formation in three groups of albino rats: 1) Intact; 2) With electrolytic lesions of neocortex over the dorsal hippocampus; and 3) with electrolytic lesions of dorsal hippocampus. The term "behavior vector" has been introduced to assess quantitatively the behavior of rats while learning. The proposed approach enables to assess active avoidance behavior in rats simultaneously by all the test parameters: 1) Reaction to the light; 2) Reaction to the electric irritation; and 3) Inter-trial spontaneous behavior. The animals were grouped by their behavioral resemblance through the learning process. The proposed method facilitates the assessment of learning capacities in animals and paves way for getting additional information concerning correlative relationships between their learning skills and other neuroethological and neurobiological parameters.
\end{abstract}

Keywords: Behavior; Learning; Memory; Active Avoidance; Hippocampus; Neocortex; Unsupervised Clustering

\section{Introduction}

Sometimes it seems difficult to generalize the outcomes of behavioral studies due to a large amount of experimental evidence on the features involved in formation of adequate behavioral strategy. Thus, a mathematical approach to the problem in general, and quantification of the measured parameters in particular, should be considered as most reasonable means to identify behavioral features and interpret obtained numeric data. Nowadays such approach is common to behavioral studies.

A wide range of mathematical methods has been proposed for the assessment of cognitive mechanisms involved in adaptive learning, repetitive decision tasks, reinforcement and strategic changes [1-5]. These references along with the related work have contributed to understanding in depth the processes underlying behavioral neuropsychology [6]. The clustering methods with different approaches and different focuses were used in studies on learning, memory and behavior [7-13]. Mathematical methods generally gained a prominent position within behavioral studies over the last centuries [1].

Accordingly, the objective of our study was to examine and confirm possibility of application of unsupervised cluster analysis algorithm for quantitative description of behavioral conformities through active avoidance acquisition in different population of albino rats $[14,15]$.
Such approach enables to assess active avoidance acquisition revealing behavioral differences and similarities among the animals within groups [16].

Efforts to reveal neuropsychological explanation to different behavioral processes were directed towards identification of brain structures involved in different types of learning and memorizing. Hippocampus represents a structure specifically related to learning and mediation of behavioral processes [17-22]. We intended to contribute to the investigation of functional significance of dorsal hippocampus in implementation of different behavioral tasks by cluster analysis.

Animals' learning abilities assessed by acquisition of active avoidance were found to vary within the test groups. Some of the animals were unable to meet learning criteria and consequently, several groups of animals with different behavioral capabilities-were identified and each group included the animals with similar behavioral features. The method of automatic classification (cluster analysis) was applied in order to extract such groups from the three populations of white rats: intact (INT); with electrolytic coagulation of neocortex above the dorsal hippocampus (NCC) and with electrolytic coagulation of dorsal hippocampus (DHPC).

The unsupervised clustering algorithm based on Parzen statistical estimation of probability density function 
for partitioning of rats according to their behavioral similarities were applied [14,15].

The term "behavior vector" for multiparameter description of behavior in learning process was introduced. The components of the vector were behavioural parameters measured for each animal and they took different numerical values during the experiment.

Such approach enables to classify the animals by their learning abilities into groups according to the degree of behavioral similarity. The behavioral parameters (features) getting different numerical values during the experiment compose the components for the behavior vector.

The observed behavioral parameters assessed experimentally were: 1 ) reactions to the light-avoidance reactions; 2) reactions to the painful foot-shock evaluated in frequencies-escape reactions; and 3) inter-trial spontaneous behavior, measured in numbers of jumping onto shelves. As all the three parameters were targeted and served to preserve from painful stimuli, we characterized the active avoidance learning by general analysis of values encompassing all the three parameters in total.

\section{Methods}

\subsection{Subjects}

Three different groups of 31 albino rats of both sexes (with an average body weight of 150 g) were examined. The animals were individually housed in stainless steel cages in a room with natural lightdark cycle and constant temperature of $20^{\circ} \mathrm{C} \pm 1^{\circ} \mathrm{C}$. The rats had free access to food and water throughout the experiment. The animals were numbered before the experiment and divided into three groups designated as Group A (intact; No. 1 - 13; n = 13), Group B (NCC; No. 41 - 49; $n=9$ ), and Group C (DHPC; No. 32 - 40; $n=9$ ).

\subsection{Apparatus}

The apparatus consisted of a chamber $(61 \times 36 \times 46)$ with three walls and a lid made of dark opaque plastic with a transparent frontal door. The floor of the chamber was made of stainless steel rods ( $2 \mathrm{~mm}$ in diameter) that were spaced $1 \mathrm{~cm}$ apart; the floor of the chamber was electrified. Dynamic shelves were attached to the lateral wall at height of $11 \mathrm{~cm}$ onto which the animals could jump up performing self-defensive behavior. The apparatus was placed in an acoustically insulated room at constant temperature of $20^{\circ} \mathrm{C} \pm 1^{\circ} \mathrm{C}$. Illumination lamp of $60 \mathrm{l} \times$ was used as a light stimulus.

\subsection{Procedure}

The scheme of the research was designed as described by Tsagareli and Djgarkava [23]. The experiment lasted 20 days with 10 trials p.d. In each trial of active avoidance conditioning the avoidance was signaled by a single light stimulus presented for 10 sec. The subjects could avoid the painful foot-shock by jumping onto the shelves. If they did not, after 10 sec on the background of condition stimulus, the foot-shock current (25 mv) was delivered for $5 \mathrm{sec}$ through the grid. The rats could escape the shock by jumping up onto the nearest shelf staying there for $3 \mathrm{sec}$ until they were forced to return to the floor. Inter- trial period was scheduled by special program (method of Monte-Carlo) that had been proposed to explore animals from different populations under identical experimental conditions; during the inter-trial intervals the rats could spontaneously jump up onto the shelves for only 3 sec. since after that the experimenter lowered the shelf and forced the animals to jump down to the floor. The grid of floor and walls of the chamber were washed with acetone, urine and/or feces were removed after completing each test.

As mentioned, three behavioral parameters were used to evaluate active avoidance conformities in albino rats: 1) jumping up onto the shelf as a response to the conditional (light) stimulus; 2) escape response to the unconditional stimulus (avoidable painful foot-shock coming through the floor); and 3) spontaneous activity (jumping onto the shelf) during the inter-trial intervals. Each experimental parameter was assessed quantitatively. The frequencies of light-induced avoidance and shock-induced escape behavior were measured for each animal tested for acquisition of active avoidance task. Inter-trial activeity was measured in numbers relevant to spontaneous jumping onto the shelves.

\subsection{Surgery}

All surgical procedures were performed under aseptic conditions. The rats were anesthetized with sodium pentobarbital (Nembutal $55 \mathrm{mg} / \mathrm{kg}$, i.p.) and placed in a stereotaxic instrument. An incision was made in the skin covering the skull and the latter was leveled. The animal had randomly received either electrolytic-induced lesions of the dorsal hippocampus or neocortex over the dorsal hippocampus. Electrolytic bilateral lesions of the hippocampus and neocortex were performed by passing a rectified current of $1.2 \mathrm{~mA}$ for $15 \mathrm{sec}$ through stainless-steel electrode $(0.2 \mathrm{~mm}$ in diameter) uninsulated at the tip (approx. $0.5 \mathrm{~mm}$ ). The lesion coordinates were identified on the basis of the rat brain stereotaxic atlas [24]. Each animal was given a 7 day recovery period before testing.

\subsection{Histology}

After completion of behavioral testing, the rats with lesions were sacrificed with an overdose of pentobarbital (100 mg/kg, i.p.) and perfused transcardially with $0.9 \%$ 
NS followed by $10 \%$ formal saline. The brains were removed and stored in $10 \%$ formal saline. The brains of all surgered rats were cut into $30 \mathrm{~mm}$-thick horizontal sections. Verifications included estimation of hippocampal and neocortical lesion extent.

\section{Experimental Data Analysis}

A wide range of statistical methods should be applied for analysis of any behavioral parameters, but besides traditional statistic methods, the paper aims to propose the cluster analysis for assessment of neuroethological data.

\subsection{Statistical Analysis}

Behavioral data were analyzed using factorial analysis of variance (ANOVA) considering lesion and daily behaveioral session as grouping factors in order to analyze the higher-order interactive effects of multiple categorical independent variables (factors) and to test for significant effects of the lesion. Additional analysis was performed using post hoc comparisons (LCD test) in cases where significant effects were found. Differences were considered to be statistically significant at $p<0.05$.

\subsection{Cluster Analysis}

Each animal placed in cabin was described by the "behavior vector" for multiparameter description of behavior in learning process. The components of the vector were behavioural parameters measured for each animal and they took different numerical values during the experiment. Consequently, several groups of animals demonstrating different behaviour along the learning process might exist.

Unsupervised clustering algorithm based on Parzen statistical estimation of probability density function has been used in order to partition the rats according to their behavior similarities. The algorithm considers the case when both the probability density of an initial data set and the number of data classes are preliminary unknown $[14,15]$. This enables to classify rats' behavior by their active avoidance acquisition ability [16].

\section{Discussion}

Data reported as the mean values \pm S.E.M of three behavioral parameters for all the three animal populations are presented in Figure 1. The curves demonstrate daily dynamics of behavioral parameters during the whole experiment $(F(2,114)=11.16, P<0.05)$.

At the early stages of the experiment it is shown that in contrast to intact or NCC animals DHPC rats are not able to perform escape behavior. During the following days the escape reaction rates are rapidly increasing and reach the criteria level on day 5 that is not observed in cases with DHPC rats-the corresponding rates remained lower for next few days.
The dynamics of avoidance behavior shows that the DHPC rats perform avoidance from the $5^{\text {th }}$ day of the experiment and despite the rates of avoidance are progresssively increasing, they remain lower than those for intact and NCC animals (Figure 1). The intact and the NCC rats reach the learning criteria level by day 13 , but the DHPC animals on day 15 (the learning criteria are accepted to be within rate ranges of 0.9 and 1 ).

When assessing inter-trial spontaneous behavior, the NCC were found to be more active than the intact rats, but the NCC were less active in comparison with the intact and the NCC animals. The pattern of dynamics was similar for all the studied groups-low at the beginning of the experiment, growing during the next few days with the following decrease of rates (Figure 1).

It is obvious that the escape frequency score significantly differs between Intact-DHPC and NCC-DHPC groups at the initial stage of the test (A), but avoidance responses score remained significantly different through the whole experiment with lower rate in DHPC rats (B); Mean value of inter-trial spontaneous activity assumes that there is statistically significant difference among all studied groups; Inter-trial behavior is statistically different in all groups at significance level $p<0.05$.

It is obvious from the curve patterns that the rats promptly escape from painful foot-shock stimulus (sharply increasing escape responses), but acquisition of avoidance reaction to the light is comparatively slow. Throughout the experiment elaboration of optimal self-defensive behavioral algorithm takes place in experimental animals: the rats begin to learn that staying on shelves helps them to avoid induced painful stimulus (foot-shock) stress and, consequently, the frequencies of avoidance responses are increasing. The variation in dynamics of inter-trial spontaneous behavior seems to be interesting - the rate of spontaneous jumping onto the shelves still remains at rather low level until the animals infer that staying on shelves is a self-defense behavior. The avoidance responses correlate with spontaneous activity causing increase of the latter (beginning from the $5^{\text {th }}$ day and lasting up to the $13^{\text {th }}$ day). On the $14^{\text {th }}$ day, after the learning criteria level has been reached in all the three studied populations, the inter-trial spontaneous activity begin to decrease and such dynamics is maintained up to the end of the experiment.

All the behavioral activities (escape, avoidance or inter-trial spontaneous activity) serve for the main strategy minimize painful stress throughout experimental treatment-to learn to jump onto the shelf. Consequently, in order to assess the roles of studied structures (dorsal hippocampus and neocortex) in learning processes the simultaneous treatment of all the three behavioral parameters and identification of common behavioral parameter are recommended. In addition, revealing individual behavioral parameters and grouping animals by their behavioral resemblance also seem desirable. 


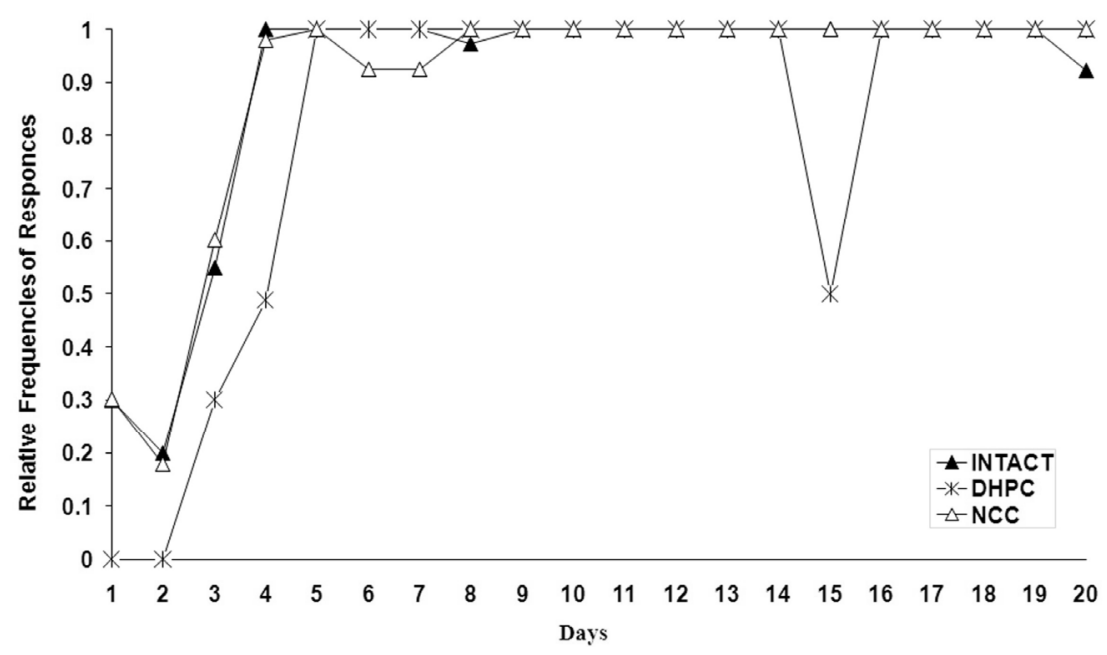

(a)

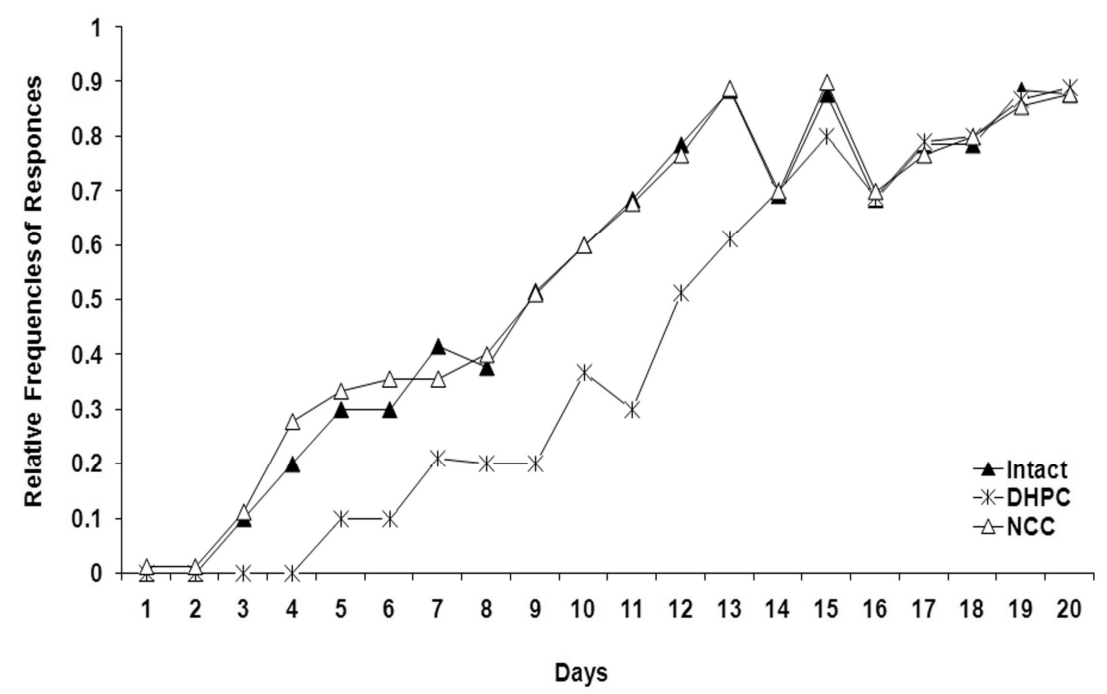

(b)

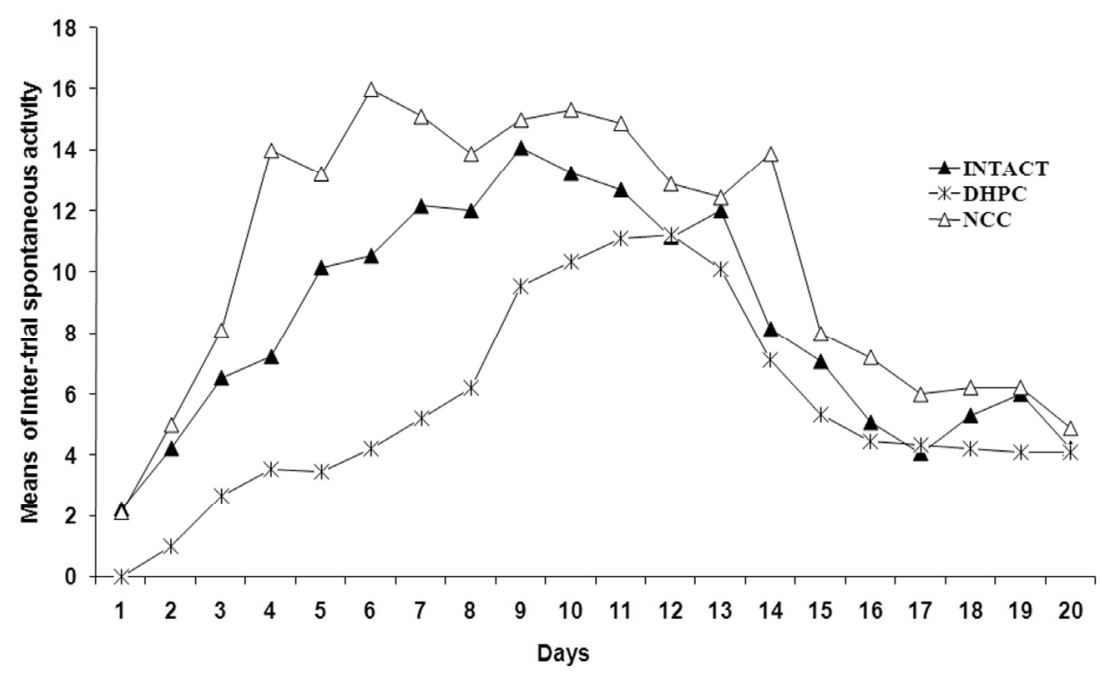

(c)

Figure 1. Dynamics of self-defensive behavior during the active avoidance memorization: (a) Escape responses to pain- ful foot-shock; (b) Avoidance responses to conditional (light) stimulus; (c) Inter-trial spontaneous activity. 
The analysis of 20-day experiment dynamics of three behavioral parameters revealed learning based adaptation conformities of animals to the aversive conditions different among the populations. The significance of differences between the studied populations obtained by factorial analysis of variance considering lesion and daily behaveioral session as grouping factors is shown on Table 1.

In case of finding significant effects additional analysis was performed using post hoc comparisons (LCD test) revealing daily differences in escape, avoidance and spontaneous behavior between the studied groups ("+"): significant differences, $p<0.05000$; “-”: no significant differences, $p>0.05000$ ) (Table 1).

However, considering differences between the mean values it seems to be the case that these differences do not reflect behavioral patterns for each individual in full. The populations involve animals with either high or low learning abilities. It should also not be excluded that the representatives of different populations might have similar learning skills. Proceeding from that, it seems significant to apply the relevant approach aimed at grouping animals by their behavioral resemblance.

Cluster analysis for classifying the animals through the learning process into classes by the degree of behavioral similarity throughout multiparameter assessment has be- en used.

Cluster analysis of experimental data involved all three groups: intact, NCC and DHPC rats (31 animals, in total). The proposed approach enabled us to assess active avoidance formation conformities in the studied groups by total analysis subjected to overall parameters. Consequently, as a result of cluster analysis, all the studied rats from different populations were classified into classes according to their behavioral similarities. Each of them included animals with similar learning abilities.

Distribution of the extracted classes obtained by use of cluster analysis of the 31 rats from different test groups was defined. The class number change dynamics (Figure 2) and relative frequencies of homogeneous classes were assessed (Figure 3).

The relative frequency of appearance of class 1 significantly differed from the other. As for the classes 2 and 3 (Figure 3), their frequencies were significantly lower than that for class 1 , however, they exceeded the other classes not included in the final analysis due to their extremely low frequencies.

The first class involved rats with most resembling behavioral patterns during active avoidance acquisition and every next class exhibited less similarity to it (Figure 3). We revealed the groups with prevalence of animals of class 1 that was assessed in percentage (Figure 4).

Table 1. The significance of differences among the studied populations obtained by factorial analysis of variance.

\begin{tabular}{|c|c|c|c|c|c|c|c|c|c|}
\hline \multirow{2}{*}{ Days } & \multicolumn{3}{|c|}{ INTACT vs. DHPC } & \multicolumn{3}{|c|}{ INTACT vs. NCC } & \multicolumn{3}{|c|}{ DHPC vs. NCC } \\
\hline & $\begin{array}{l}\text { Escape } \\
\text { Behavior }\end{array}$ & $\begin{array}{l}\text { Avoidance } \\
\text { Behavior }\end{array}$ & $\begin{array}{c}\text { Spontaneous } \\
\text { Behavior }\end{array}$ & $\begin{array}{l}\text { Escape } \\
\text { Behavior }\end{array}$ & $\begin{array}{c}\text { Avoidance } \\
\text { Behavior }\end{array}$ & $\begin{array}{c}\text { Spontaneous } \\
\text { Behavior }\end{array}$ & $\begin{array}{l}\text { Escape } \\
\text { Behavior }\end{array}$ & $\begin{array}{l}\text { Avoidance } \\
\text { Behavior }\end{array}$ & $\begin{array}{c}\text { Spontaneous } \\
\text { Behavior }\end{array}$ \\
\hline 1 & + & - & - & - & - & - & + & - & - \\
\hline 2 & + & - & + & - & - & - & + & - & + \\
\hline 3 & + & + & + & - & - & - & + & + & + \\
\hline 4 & + & + & + & - & + & + & + & + & + \\
\hline 5 & - & + & + & - & - & + & - & + & + \\
\hline 6 & - & + & + & + & + & + & + & + & + \\
\hline 7 & - & + & + & + & + & + & + & + & + \\
\hline 8 & - & + & + & - & - & - & - & + & + \\
\hline 9 & - & + & + & - & - & - & - & + & + \\
\hline 10 & - & + & + & - & - & - & - & + & + \\
\hline 11 & - & + & - & - & - & - & - & + & + \\
\hline 12 & - & + & - & - & - & - & - & + & - \\
\hline 13 & - & + & - & - & - & - & - & + & - \\
\hline 14 & - & - & - & - & - & + & - & - & + \\
\hline 15 & + & + & - & - & - & - & + & + & - \\
\hline 16 & - & - & - & - & - & - & - & - & - \\
\hline 17 & - & - & - & - & - & - & - & - & - \\
\hline 18 & - & - & - & - & - & - & - & - & - \\
\hline 19 & - & - & - & - & - & - & - & - & - \\
\hline 20 & - & - & - & - & - & - & - & - & - \\
\hline
\end{tabular}




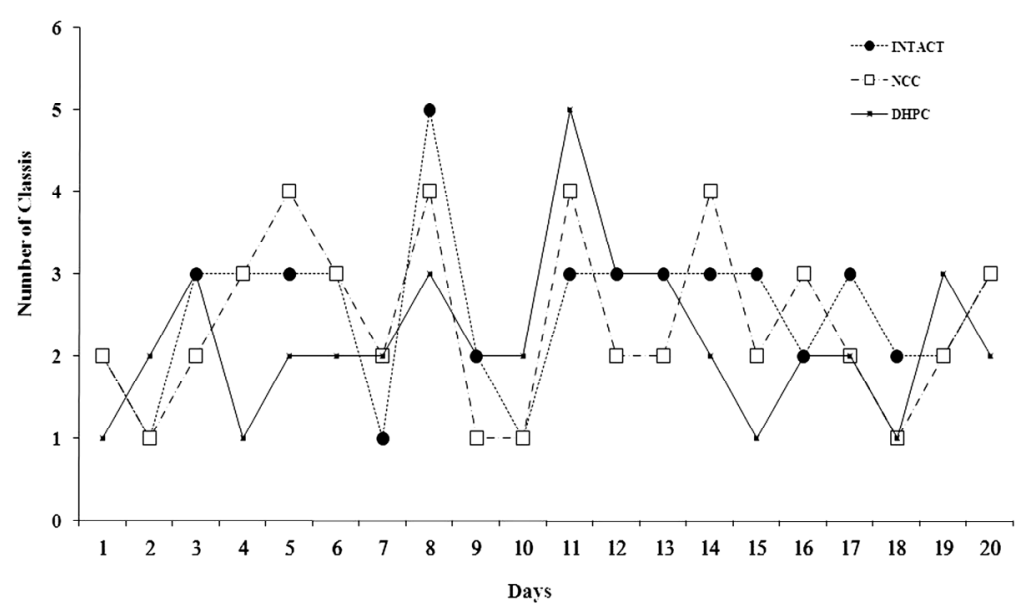

Figure 2. The class number change dynamics in the course of 20 day.

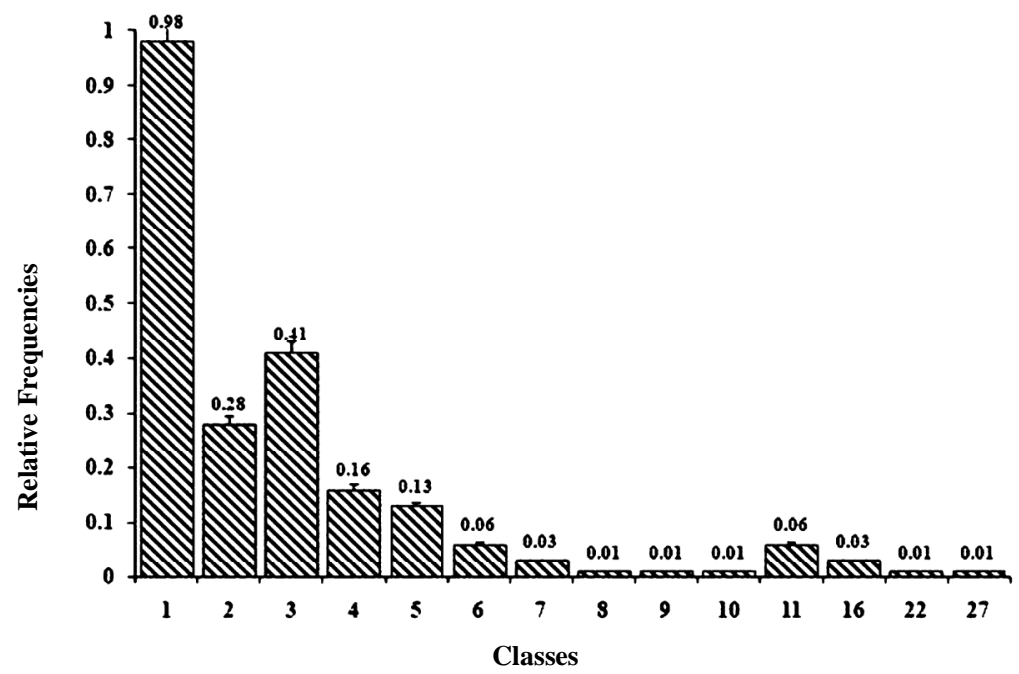

Figure 3. Relative frequencies of different classes defined by cluster analysis.

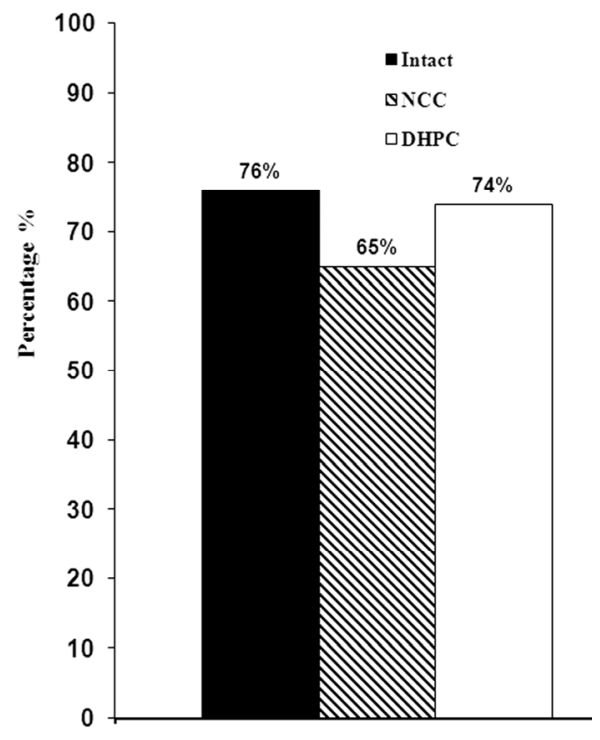

Figure 4. Percentage of the animals involved in class 1 from different test groups.
Proceeding from the analysis of experimental data, it was established that $24 \%$ of intact, $35 \%$ of NCC and $26 \%$ of DHPC rats were not included in class 1 (Figure 4).

Therefore, in order to assess quantitatively individual learning abilities and taking into account the fact that large majority of the animals were referred to class 1 , the rats appearance frequency in class 1 (frequency range 0 - 1) was conditionally divided into four frequency intervals (0.90 - 1; 0.80 - 0.89; 0.70 - 0.79; 0.60 - 0.69).

The number of the appearances in class 1 has been divided by the number of days in order to calculate the appearance frequency for each rat. This enabled us to identify the mixed groups each containing the animals with different learning abilities of active avoidance through 20 day experiment. Distribution of the tested animals (numbered) within the frequency intervals is shown on Table 2.

The frequency interval of 0.9 - 1 included only the rats being most successful by their active avoidance behavior - superior learners (3 intact rats). The animals of the second rate group-good learners $(0.8-0.89)$ included the 


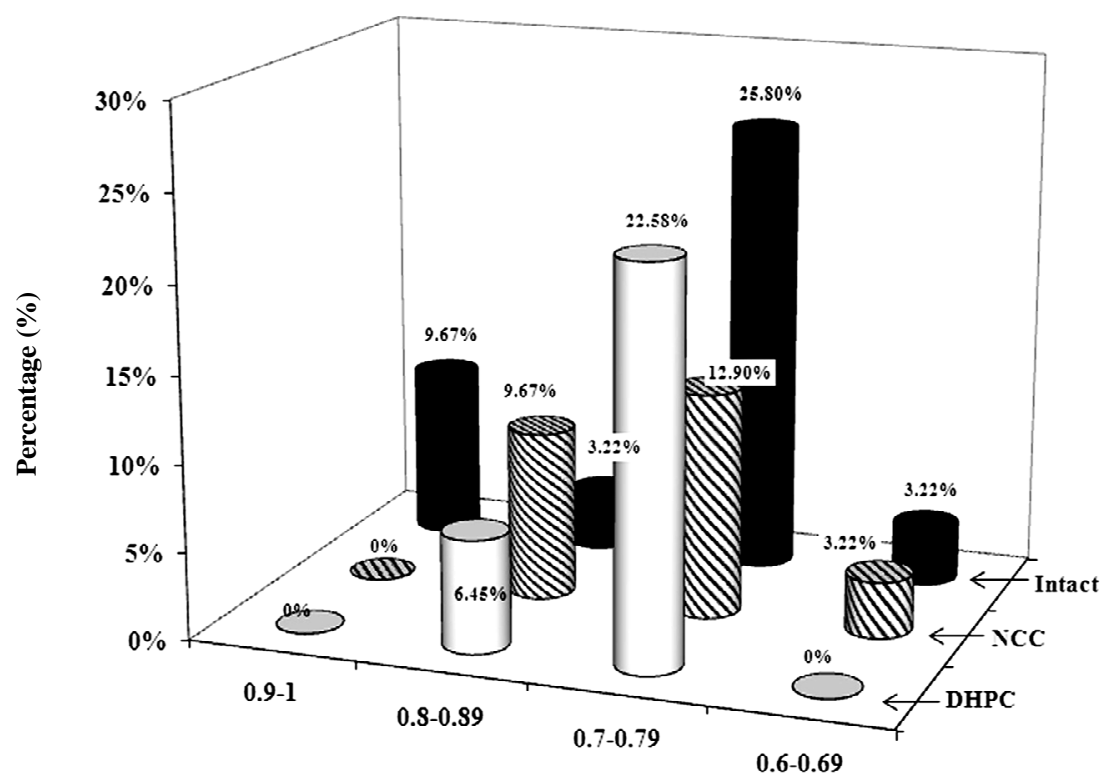

Figure 5. Distribution of the animals (class 1) with different learning abilities in divided frequency intervals.

Table 2. Distribution of tested animals (numbered) within the frequency intervals.

\begin{tabular}{ccccc}
\hline \multirow{2}{*}{ Groups } & \multicolumn{4}{c}{ Frequency Ranges } \\
\cline { 2 - 5 } & $0.9-1$ & $0.8-0.89$ & $0.7-0.79$ & $0.6-0.69$ \\
\hline INTACT & $2,7,11$ & 8 & $1,3,4,5,6,10,12,13$ & 9 \\
NCC & 0 & $41,42,43$ & $45,46,47,48,49$ & 44 \\
DHPC & 0 & 32,38 & $33,34,35,36,37,39,40$ & 0 \\
\hline
\end{tabular}

ones with well performed behavior test (1 intact; 3 NCC and 2 DHPC rats). However, they were less successful than the animals of group 1 . Medium learners -8 intact, 5 NCC and 7 DHPC were within the interval of 0.7 0.79 . The fourth rate interval-inferior learners (0.6 $0.69)$ contained only 1 intact and 1 NCC rats. No DHPC rats were found to meet the criteria stipulated for that group.

Percentage of animals from each population included in class 1 was established. Out of all the studied populations, $9.67 \%$ of intact animals' best succeeded at active avoidance behavior. No animals among DHPC or NCC groups could achieve such levels. 3.22\% of intact, $9.67 \%$ of NCC and $6.45 \%$ DHPC rats were found to be good at learning. Lower learning ability was revealed among $25.8 \%$ of intact, $12.9 \%$ of NCC and $6.45 \%$ with DHPC (Figure 5).

\section{Conclusion}

The proposed approach enables assessment of active avoidance behavior in rats by analysis of three or more parameters in total. It enables further grouping of all the stu- died animals from different populations by their behaveioral similarities. Besides, the proposed approach is suited for the assessment of learning capacities of animals. It also facilitates getting additional information and defining correlation between the learning skills and other neuroethological and neurobiological parameters.

\section{REFERENCES}

[1] K. Hausken and J. F. Moxnes, "Behaviorist Stochastic Modeling of Instrumental Learning," Behavioural Processes, Vol. 56, No. 2, 2001, pp. 121-129. doi:10.1016/S0376-6357(01)00192-9

[2] C. Kolodziejski, B. Porr and F. Wörgötter, "Mathematical Properties of Neuronal TD-Rules and Differential Hebbian Learning: A Comparison,” Biological Cybernetics, Vol. 98, No. 3, 2008, pp. 259-272. doi:10.1007/s00422-007-0209-6

[3] G. McCollum, "Mathematics Reflecting Sensorimotor Organization,” Biological Cybernetics, Vol. 88, No. 2, 2003, pp. 108-128. doi:10.1007/s00422-002-0344-z

[4] M. P. Paulus and M. A. Geyer, "Quantitative Assessment of the Microstructure of Rat Behavior: I. $f(d)$, the Extension of the Scaling Hypothesis," Psychopharmacology, Vol. 113, No. 2, 2005, pp. 177-186. doi:10.1007/BF02245695

[5] P. E. Rapp, "Quantitative Characterization of Animal Behavior Following Blast Exposure,” Cognitive Neurodynamics, Vol. 1, No. 4, 2007, pp. 287-293. doi:10.1007/s11571-007-9027-8

[6] S. Ito, H. Yuasa, Z. Luo, M. Ito and D. Yanagihara, “A Mathematical Model of Adaptive Behavior in Quadruped Locomotion,” Biological Cybernetics, Vol. 78, No. 5, 1998, pp. 337-347. doi:10.1007/s004220050438

[7] M. C. Baker and D. M. Logue, "Population Differentiation 
in a Complex Bird Sound: A Comparison of Three Bioacoustical Analysis Procedures,” Ethology, Vol. 109, No. 3, 2003, pp. 223-242. doi:10.1046/j.1439-0310.2003.00866.x

[8] D. Balslev, F. A. Nielsen, S. A. Frutiger, J. J. Sidtis, T. B. Christiansen, C. Svarer, S. C. Strother, D. A. Rottenberg, L. K. Hansen, O. B. Paulson and I. Law, "Cluster Analysis of Activity-Time Series in Motor Learning," Human Brain Mapping, Vol. 15, No. 3, 2002, pp. 135-145. doi:10.1002/hbm.10015

[9] H. Cohen, J. Zohar, M. A. Matar, Z. Kaplan and A. B. Geva, "Unsupervised Fuzzy Clustering Analysis Supports Behavioral Cutoff Criteria in an Animal Model of Posttraumatic Stress Disorder,” Biological Psychiatry, Vol. 58, No. 8, 2005, pp. 640-650. doi:10.1016/j.biopsych.2005.04.002

[10] P. Edison, H. A. Archer, A. Gerhard, R. Hinz, N. Pavese, F. E. Turkheimer, A. Y. F. T. Hammers, N. Fox, A. Kennedy, M. Rossor and D. J. Brooks, "Microglia, Amyloid, and Cognition in Alzheimer's Disease: An [11C] (R) PK11195-PET and [11C] PIB-PET Study,” Neurobiolpgy of Disease, Vol. 32, No. 3, 2008, pp. 412-419. doi:10.1016/j.nbd.2008.08.001

[11] C. Lochner, S. M. J. Hemmings, C. J. Kinnear, D. Nel, S. Seedat, J. C. Moolman-Smook and D. J. Stein, "Cluster Analysis of Obsessive-Compulsive Symptomatology: Identifying Obsessive-Compulsive Disorder Subtypes,” Israel Journal of Psychiatry and Related Sciences, Vol. 45, No. 3, 2008, pp. 164-176.

[12] J. C. McDonagh, R. B. Gorman, E. E. Gilliam, T. G. Hornby, R. M. Reinking and D. G. Stuart, "Properties of Spinal Motoneurons and Interneurons in the Adult Turtle: Provisional Classification by Cluster Analysis,” The Journal of Comparative Neurology, Vol. 400, No. 4, 1998, pp. 544-570. doi:10.1002/(SICI)1096-9861(19981102)400:4<544::AID -CNE8>3.0.CO;2-A

[13] M. C. Stevens, D. A. Fein, M. Dunn, D. D. Allen, L. H. Waterhouse, C. M. D. Feinstein and I. M. D. Rapin, "Subgroups of Children with Autism by Cluster Analysis: A Longitudinal Examination," Journal of the American Academy of Child \& Adolescent Psychiatry, Vol. 39, No. 3, 2000, pp. 346-352. doi:10.1097/00004583-200003000-00017

[14] O. Tavdishvili, "Automatic Classification Algorithm for Observable Data Set," Proceedings of the Institute of Cybernetics, Vol. 3, No. 1-2, 2004, pp. 136-141.
[15] O. Tavdishvili and T. Sulaberidze, "Segmentation Method of 3D Segments Extraction on the Scene Image,” In: J. M. Blackledge and M. J. Turner, Eds., Image Processing III: Mathematical Methods, Algorithms and Applications, Horwood Publishing, Chichester, 2001, pp. 82-88.

[16] O. Tavdishvili, N. Archvadze, S. Tsagareli, A. Stamateli and M. Gvajaia, "The Study of Rats' Active Avoidance Behavior by the Cluster Analysis,” Life System Modeling and Intelligent Computing, Vol. 6330, 2010, pp. 180-188.

[17] J. Ferbinteanu and M. L. Shapiro, "Prospective and Retrospective Memory Coding in the Hippocampus," Neuron, Vol. 40, No. 6, 2003, pp. 1227-1239. doi:10.1016/S0896-6273(03)00752-9

[18] K. Henke, V. Treyer, E. T. Nagy, S. Kneifel, M. Dursteler, R. M. Nitsch and A. Buckb, "Active Hippocampus during Nonconscious Memories," Consciousness and Cognition, Vol. 12, No. 1, 2003, pp. 31-48. doi:10.1016/S1053-8100(02)00006-5

[19] J. Ji, and S. Maren, "Electrolytic Lesions of the Dorsal Hippocampus Disrupt Renewal of Conditioned Fear after Extinction,” Learning Memory, Vol. 12, No. 3, 2005, pp. 270-276. doi:10.1101/lm.91705

[20] K. Longden, "Constraining the Function of CA1 in Associative Memory Models of the Hippocampus,” Ph.D. Thesis, University of Edinburgh, Edinburgh, 2005.

[21] I. Martınez, G. L. Quirarte, S. Diaz-Cintra, C. Quiroz and R. A. Prado-Alcala, "Effects of Lesions of Hippocampal Fields CA1 and CA3 on Acquisition of Inhibitory Avoidance,” Neuropsychobiology, Vol. 46, No. 2, 2002, pp. 97-103. doi:10.1159/000065419

[22] D. Schulz, J. P. Huston, K. Jezek, H. L. Haas, A. RothHarer, O. Selbach and H. J. Luhmann, "Water Maze Performance, Exploratory Activity, Inhibitory Avoidance and Hippocampal Plasticity in Aged Superior and Inferior Learners,” European Journal of Neuroscience, Vol. 16, No. 11, 2002, pp. 2175-2185. doi:10.1046/j.1460-9568.2002.02282.x

[23] S. Tsagareli and N. Djgarkava, "The Machine Processing of Experimental Results of the Formation and Maintenance of Avoiding and Feeding Habits,” In: R. Zhordania, Ed., Biology and Contemporaneity, Tbilisi University Press, Tbilisi, 2002, pp. 166-177.

[24] G. Paxinos and C. Watson, "The Rat Brain in Stereotaxic Coordinates,” 3rd Edition, Academic Press, San Diego, 1997. 